\title{
Liquid-Phase Electron Microscopy in Structural Protein Studies
}

Gabriel Ing ${ }^{1,2}$, Cesare de Pace ${ }^{1,2}$, Silvia Acosta Guitierrez ${ }^{1,2}$, Gabrielle Marchello ${ }^{1,2}$, Simona Pilotto ${ }^{2}$, Diana Leite $^{1}$, Neil Wilkinson ${ }^{3}$, Francesco L. Gervasio ${ }^{4}$, Finn Werner $^{2}$, Lorena Ruiz-Perez ${ }^{1,2}$ and Giuseppe Battaglia ${ }^{1,2,5,6}$

${ }^{1}$ Department of Chemistry, University College London, UK.

${ }^{2}$ Institute of Structural and Molecular Biology, University College London, UK.

${ }^{3}$ University of Geneva, Pharmaceutical Sciences, Geneva, Switzerland.

${ }^{4}$ Gatan, UK.

${ }^{5}$ Institute for Bioengineering of Catalunya (IBEC), The Barcelona Institute of Science and Technology, Barcelona, Spain.

${ }^{6}$ Catalan Institution for Research and Advanced Studies (ICREA), Barcelona, Spain.

The use of transmission electron microscopy (TEM) has revolutionized structural biology through developments in cryogenic TEM (cryo-EM) [1]. This method is exploding in popularity due to its ability of imaging at high resolution large protein complexes, including proteins found in cellular membranes. Cryo-EM for structural biology uses computational algorithms to produce 3D reconstructions of the protein complexes. This is done employing a high number $\left(>10^{5}\right)$ of individual protein projections from TEM images of vitrified samples. While this field has many applications for molecular biology and can image the proteins close to their native state, vitrification of the sample is not quite the native state of the protein. Furthermore, the protein is frozen and therefore static so dynamic information can only be inferred from the differences in the populations of protein particles. With this shortcoming in mind, we aimed to use liquid-phase transmission electron microscopy (LTEM) for structural and dynamic studies of proteins.

To investigate proteins in solution, we have used liquid holders developed by DENS solutions for liquid phase transmission electron microscopy [2]. We have studied three different proteins by LTEM with two different aims. Firstly, we have explored the structural dynamics of two different proteins using cryo-EM $3 \mathrm{D}$ reconstruction algorithms, whilst also biasing the reconstruction process with models extracted from a molecular dynamics simulation. By doing so we are able to screen some of the low resolution features of the investigated proteins, therefore showing that our method is viable to study protein structure and dynamics within their native solution state. For this work we have first used apoferritin (Figure 1), a cryoEM standard which has a spherical cage-like structure with an outer radius of $12 \mathrm{~nm}$ and a high level of symmetry [3]. As second case we have used archaeal RNA polymerase, an asymmetric protein with a crab-claw like shape with a stalk and a DNA binding cleft (Figure 2) [4]. Our results capture the protein shape and some features and are available as a preprint (ref).

Here we also present some preliminary results of a project to image the aggregation of amyloid-beta protein, which is highly associated with Alzheimer's disease. As LTEM images the proteins in solution, it is possible to image ongoing processes occurring, including the aggregation of monomers into larger structures. Our early results, including Figure 3, show potential for LTEM to visualize the protein aggregation pathway on a structural level. This information would have important clinical significance for the study of neurodegenerative diseases caused by protein misfolding and aggregation.

We believe our findings on the use of LTEM for biological systems and in particular protein can very well complement Cryo EM studies offering an extra temporal dimension. Moreover, these results offer an exciting insight into the future potential molecular biology studies by LTEM. 


\section{References:}

[1] X Bai, G McMullan and SHW Scheres, Trends in Biochemical Sciences 40(1) (2015), p. 49.

[2] JT van Omme et al, Journal of Materials Chemistry 8(1) (2020), p. 10781.

[3] EC Theil, Nanotechnol. Percept. 8(1), (2012) p. 7.

[4] T Fouqueau et al, Emerging Topics in Life Sciences 2(4) (2018), p. 517.

[5] C De Pace et al, bioRxiv (2021), p. 2021.04.30.442083.

[6] GF Chen et al, Acta Pharmacologica Sinica 38(9) (2017), p. 1205.
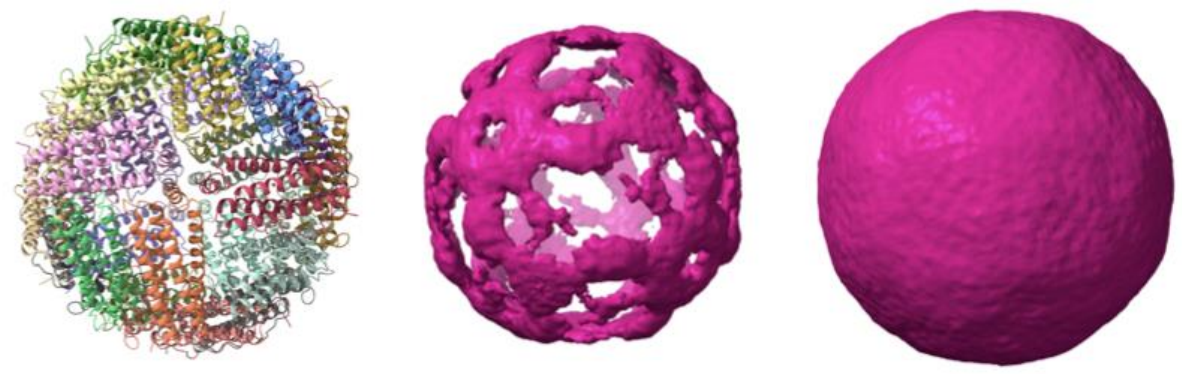

Figure 1. Reconstructions of apoferritin from LTEM videos. A reconstructed volume of apoferritin is shown at two different threshold levels with the crystal structure shown on the left for comparison (PDB ID: 1MFR).
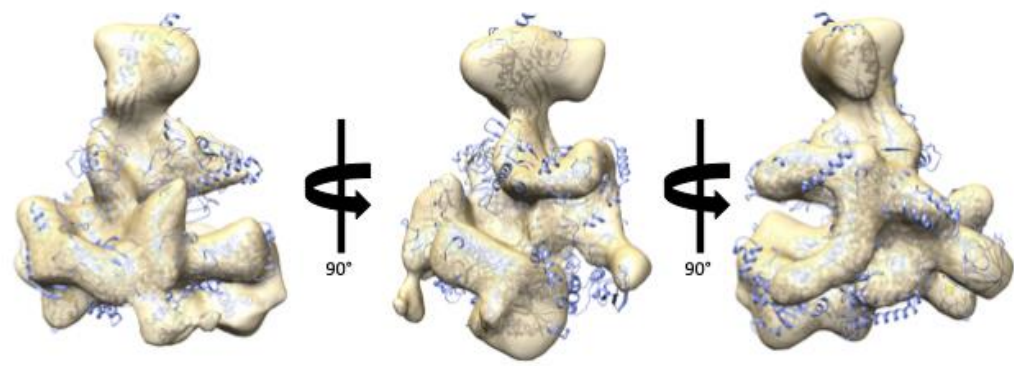

Figure 2. RNA polymerase reconstruction from LTEM with a known structural model fitted inside (blue)

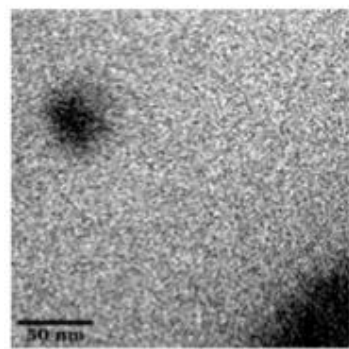

Os

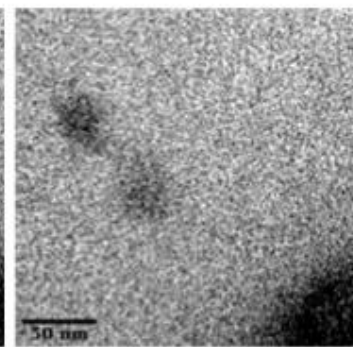

$2 \mathrm{~s}$

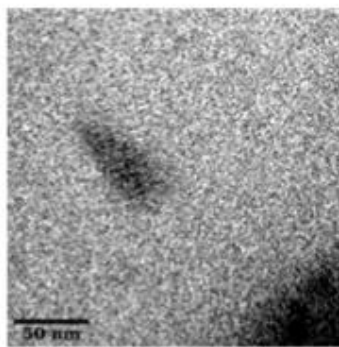

$3 s$

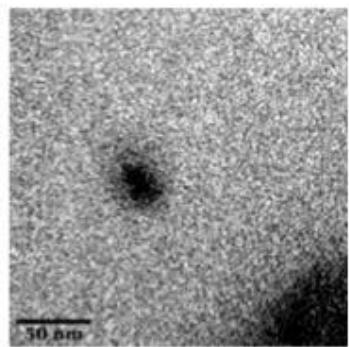

$5 s$

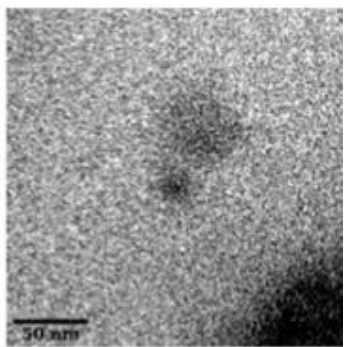

$7 s$

Figure 3. Amyloid-Beta aggregate evolving over time in solution by LTEM. 